\title{
TRANSLITERATION AND TRANSLATION
}

IN THE TRANSLATION of the text, Bengali terms are preserved wherever adequate English translations have not been found, and the terms are explained in the footnotes. In such cases, as well as throughout the transliteration of the text itself, we have tried to use the system of transliteration accepted by most scholars for the Sanskritic languages. However, Bengali is peculiar among the Sanskritic languages in certain respects. For example, the labio-dental spirant (represented by the letter $v$ ) does not occur in Bengali. Where $v$ occurs in Sanskrit, $b$ occurs in Bengali; therefore, $b$ has been used in our transliteration, except where a cluster of consonant and $v$ occurs or where the reference is to a Sanskrit, not a Bengali, word. Furthermore, the pronunciation of the Bengali symbol usually transliterated by $y$, when it occurs medially in a word, depends upon the vowel which precedes it. If this symbol is preceded by $o$ or $u$, and sometimes when it is preceded by $a$ ([j]), it is pronounced as $w$; we have thus indicated this symbol in our transliteration when it occurs in the above positions as $(w)$. Elsewhere, we have tried to be consistent with custom, even to the extent of preserving sentence final -a, which is not pronounced in reading Bengali poetry.

A remark or two might be made about the scribal peculiarities of the manuscript, which have some bearing on our transliteration of the text. The scribe's hand does not always distinguish between the characters represented in our transliteration by $l$ and $n$, nor does he usually distinguish between what we have represented as $b$ and $r$. The $l-n$ variation may be indicative of dialect or merely of the similarity of the characters in Bengali script; the $b-r$ varia- 
viii

tion is clearly scribal peculiarity. In any case, in both situations we have read the variant appropriate to the context, and have represented it as such in our transliteration.

In some cases, Bengali dialectical or other nonstandard forms occur in the manuscript of the text. Such are given in our translation in the form in which they occur in the manuscript, with the more standard form being given in the notes wherever possible. For example, the form ukil will be left as such in our translation, with a note which refers to the more standard form vakil.

Place names are given in the translation in the form in which they occur in the text, with the Anglicized form (where possible) either in following brackets or in the notes, e. g., Bardhamān [Burdwan], Palāsi [Plassey]. In the Introduction to the text, however, place names are given throughout in Anglicized form, e.g., Vishnupur.

We have tried to make the translation of the text as accurate as readability in English will allow. In some places, the manuscript is indecipherable due to mutilation or faded ink, or the reading is unclear for some other reason. Such places, as well as those at which the manuscript reading is at variance with the printed text, are indicated in the notes. 